\title{
Novel Horn Designs for Power Ultrasonics
}

\author{
S. Sherrit, M. Badescu, X. Bao, Y. Bar-Cohen, Z. Chang, \\ Jet Propulsion Laboratory, \\ California Institute of Technology, Pasadena, CA \\ email: ssherrit@jpl.nasa.gov
}

\begin{abstract}
Ultrasonic horns are used in a variety of industrial and medical applications. At JPL a rock-sampling tool based on an ultrasonic horn was developed to drill, abrade and core rock samples including hard basalts. This device is an impact device, which uses ultrasonic vibrations that occur at the horn tip to produce a sonic resonance with the aid of a loosely connected mass. Although standard horns are found in many current industrial designs they suffer from a few key limitations when used for USDC applications. Manufacturing a horn requires turning down stock material (e.g. Titanium) from the larger outer diameter to the horn tip diameter, and this process is both time consuming and wasteful. In this paper, we present novel horn designs that are specifically designed for impact applications as the USDC. One such design addressed the excessive length that is involved with the use of the horn limiting its applications when system dimensions are constrained. For this purpose, a folded horn design was conceived that reduces the overall length of the resonator (physical length) but maintains or increases the acoustic length. Initial experiments with horns having such a design indicate that the tip displacement can be further adjusted by phasing the bending displacements and the extensional displacements. Another conceived horn design is the "dog bone" horn that uses an end mass on the horn tip to increase the impact efficiency of the horn. In this paper, the experimental results for these novel horn designs are presented and compared to the results predicted by theory.
\end{abstract}

Keywords; piezoelectric, ultrasonic horn, drilling, high power

\section{INTRODUCTION}

A variety of industrial applications exist where power ultrasonic elements such as the ultrasonic horn are used. These include the Automotive, Food Preparation, Medical, Textile and Material Joining and Fabrication Industries. The standard transducer used in these devices consists of three main parts, the backing, the piezoelectric elements and the horn. The horn acts as a strain amplifier and for a stepped horn the strain amplification is a function of the square of the diameter ratio. The horn length primarily determines the frequency of resonance and the level of amplification at the resonance is proportional to the transducer mechanical $\mathrm{Q}$, which is a function of the attenuation of the horn material and radiation damping.

A drilling/coring device was developed at JPL and Cybersonics Inc. that is based on the ultrasonic horn and a schematic of the ultrasonic/sonic driller/corer (USDC) is shown in Figure 1 along with a photograph of a device operating while being held by the power cord. The device consists of three main parts; an ultrasonic actuator, a free mass and a drill stem. Circuit analysis of the actuator based on the equivalent circuit of a piezoelectric and acoustic elements as discussed by Mason[1],[2] was reported previously[3]. This particular standard stepped horn [4] design was found to have a resonance at $21.5 \mathrm{kHz}$ and the free tip velocity at resonance was determined to be linear with respect to the applied voltage and ranged from 1 to $10 \mathrm{~m} / \mathrm{s}$ depending on the acoustic load.

The vibrations of the horn tip excite the free mass, which resonates between the horn tip and the top of the drill stem at frequency of the order of $1000 \mathrm{~Hz}$ depending on the axial force. Acoustic energy in the free mass resonator is transferred to the top of the drill stem and propagates to the bit/rock interface where the rock is excited past its ultimate strain and fractures. A complete model of this design was recently published [5] which predicted the drilling rates as a function of the drive power. In order to increase the performance of the drill and make the design more compact we have investigated the "dog-bone" and "folded" horn designs. Schematic diagrams of these novel horn designs are shown in Figure 2.
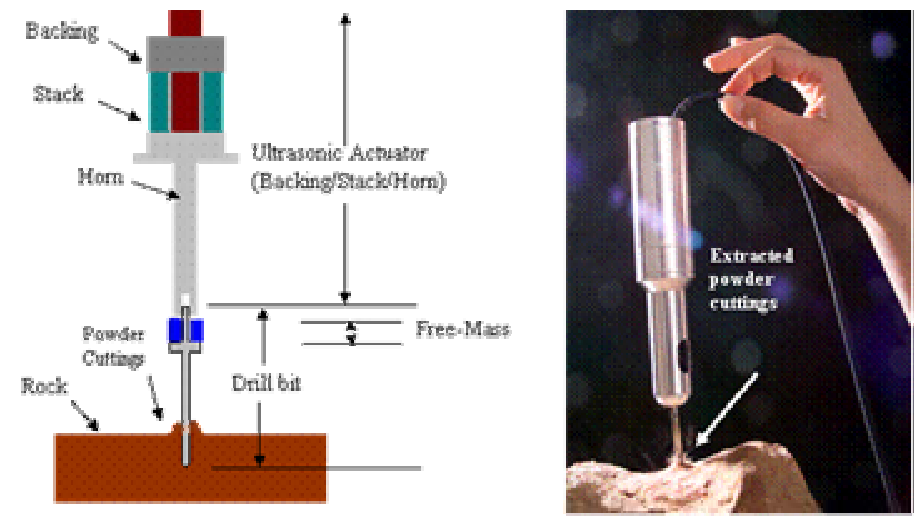

Figure 1. Photograph and Schematic of the Ultrasonic/Sonic Driller/Corer.

\section{FOLDED HORNS}

In many applications it would be useful to reduce the resonance frequency which generally requires the use of longer horns. Though the horn can be of the order of fractions of meters long, in many applications size is of a premium for space applications. In addition, manufacturing a horn such as shown in Figure 1 requires the turning down of the stock titanium from the larger outer diameter to the horn tip diameter, which is both time consuming and wasteful. In the following section we present our analysis on a variety of new horn 
designs and compare the results to initial experimental measurements

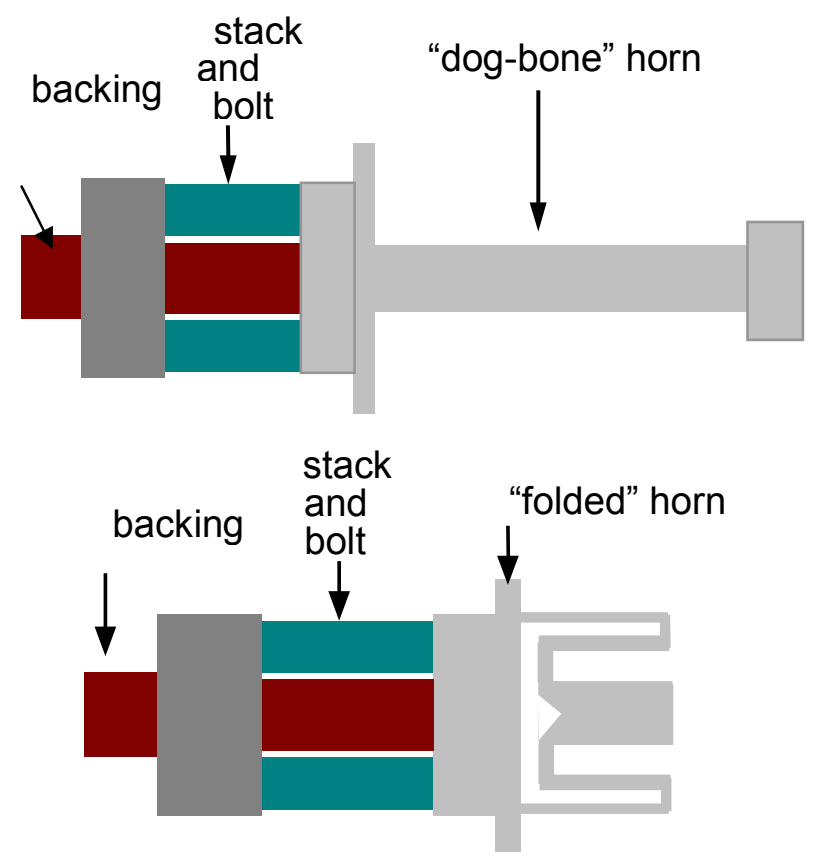

Figure 2. A "dog-bone" horn.(top) and an inverted stepped horn with three steps(bottom).

In a folded horn as is shown in Figure 2 the tip displacement can be further adjusted by including bending displacements. By adjusting the fold thickness one can increase or decrease the bending contributions to the tip displacement. This gives transducer designers another degree of freedom in the horn design in addition to the number of folds. The inverted horn is similar to the standard horn however the horn tip is a tube rather than a solid rod. In the doubly folded horn the horn starts out as an inverted horn with the same area ratio. (cross sectional area of the shell is the same as the cross sectional area of the horn tip of Figure 1.) At approximately $1 / 3$ the length of the standard horn the shell is folded back towards the base and the thickness of this length of shell is adjusted to maintain the same area ratio. Finally as the horn approaches the base it is turned once again to form a solid tip. In order to investigate the one-dimensional model a series of FEM modal (ANSYS[6]) and harmonic simulations were performed on inverted, single and double folded horns. An axis-symmetric view of the models is shown in Figure 3. The inverted and folded horns are designed to keep the acoustic length and the cross sectional areas the same as those for the standard horn. It should be noted that we have shown only inverted folded horns however one can visualize regular folded horns and combinations of the two folded horn types connected mechanically in series. The axis of rotation runs vertically on the left side of the devices shown. Table 1 lists the horn type and the $1^{\text {st }}$ length extensional resonance frequency determined from the ANSYS modal analysis. The data shows an overall reduction of the resonance frequency as folds are added, The resonance frequency was also shown to increase as the fold thickness increased. In simulations showing the displacements tip displacement was also shown to increase as the fold thickness decreased due to an increase in bending
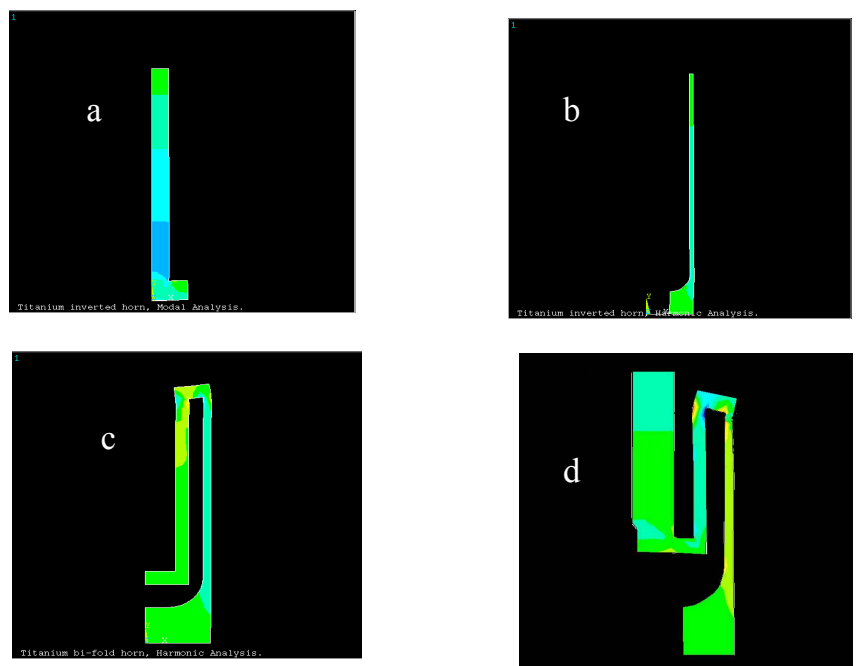

displacements.

Figure 3. An ANSYS FEM output using an axis-symmetric mesh of the a) regular stepped horn, b) inverted stepped horn, c) inverted stepped horn with 1 fold and d) the inverted stepped horn with 2 folds.

Table 1. A list of the resonance frequencies for the various horn types is shown in kHz. Acoustic length and cross sectional area is kept constant to first order. The frequency is also shown as a function of the thickness of the fold.

\begin{tabular}{lc}
\hline Horn Type & $\begin{array}{c}\text { Resonance } \\
\text { Frequency } \\
(\mathrm{kHz})\end{array}$ \\
\hline Regular $\mathrm{R}$ & 18.3 \\
Inverted no folds $\mathrm{IF}_{0}$ & 18.3 \\
Inverted one folds $\mathrm{IF}_{1}(2 \mathrm{~mm}$ thick fold $)$ & 14.7 \\
Inverted one folds $\mathrm{IF}_{1}$ (4 mm thick fold $)$ & 16.0 \\
Inverted one folds $\mathrm{IF}_{1}(6 \mathrm{~mm}$ thick fold $)$ & 16.0 \\
Inverted two folds $\mathrm{IF}_{2}(2 \mathrm{~mm}$ thick fold $)$ & 14.1 \\
Inverted two folds $\mathrm{IF}_{2}(4 \mathrm{~mm}$ thick fold $)$ & 16.2 \\
Inverted two folds $\mathrm{IF}_{2}(6 \mathrm{~mm}$ thick fold $)$ & 16.4 \\
\hline
\end{tabular}

The FEM results on the folded horn displayed a variety of behaviors including extension in the inner and outer sections as well as the midsection of the horn. The fold width was found to affect the resonance frequency of the device below a few millimeters, which was likely due to bending at the fold. This suggested that the fold thickness can be used to fine-tune the resonance of the device. In order to test the validity of the FEM results a folded horn was fabricated as shown in Figure 4. It was apparent from the solid models that manufacturing a 
horn as is shown in Figure $3 \mathrm{c}$ and $3 \mathrm{~d}$ from titanium could not be easily accomplished using standard manufacturing techniques and the horn shown in Figure 4 was fabricated in two parts using EDM.

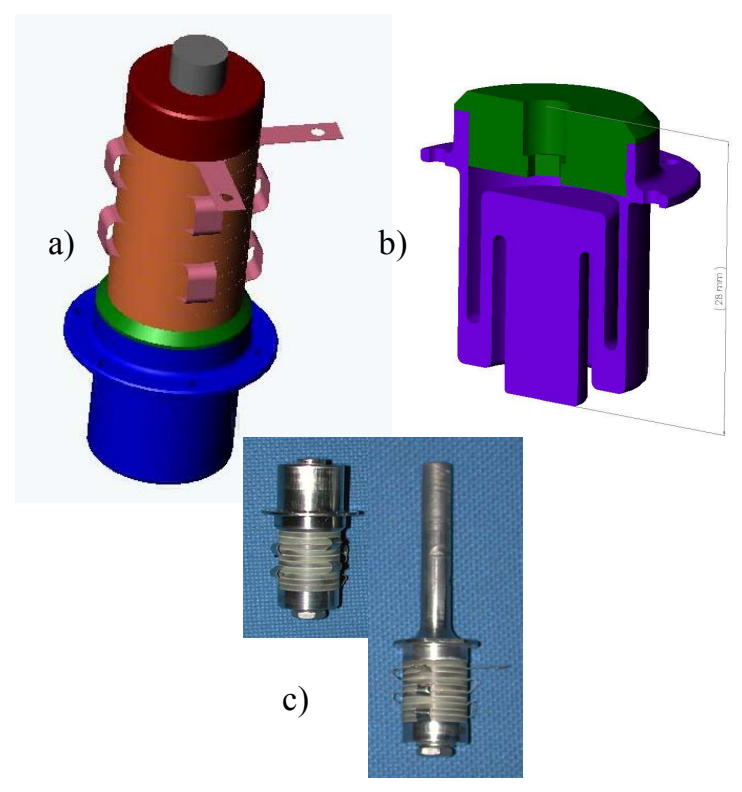

Figure 4. A Solidworks assembly drawing of the (a) folded horn, piezoelectric stack actuator, and backing. (b) Cross section of the folded horn. c) A photograph of assembled folded horn compared to standard stepped horn.

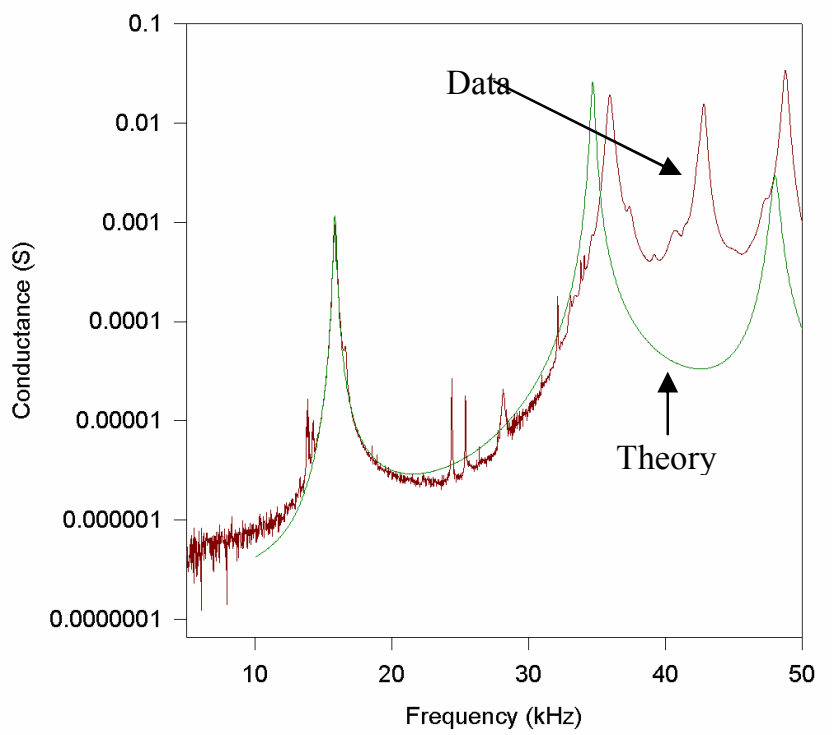

Figure 5. The impedance spectra of the folded horn compared to the standard model. In order to match the amplitude of the fundamental peak the quality of the horn material had to be reduced by a factor of 10 . The reduced resonance amplitude in the impedance values for the folded horn is the result of the lower $\mathrm{Q}$ of the device, which is likely caused by poor energy transfer across the screw threads in the base.
The impedance spectrum of the folded horn is shown in Figure 5. The mechanical Q of the folded horn was a factor of ten below the standard straight horn. This had a detrimental effect on the displacement measured at the tip of the folded horn. It was also a factor of 10 roughly smaller (10 microns as compared to $>100)$ than the straight horn. Upon closer inspection of this folded horn design it was determined that the screw threads connecting the base plate of the horn to the outer walls of the horn were the likely source of the increased dissipation of energy and reduction of resonance. Another design without screw threads that can be manufactured quite easily, which we intend to investigate was reported earlier[7].

\section{IMPACT HORNS}

The USDC is an impact device which converts ultrasonic or sonic energy to lower frequency pulses. The device efficiency is related to the amplitude and the momentum transfer of the tip to the free mass which is a function of the horn design and impact area. The interaction time of the tip with the free mass is limited to 10's of microseconds so only the first few centimeters of the horn experience the reaction force while the free mass is in contact. This suggests that for an impact device we may increase the amplitude of the device without reducing the effective interaction mass by increasing the horn diameter only at the horn tip. We studied 5 different horn designs including: the conventional horn with a crosssection area reduction, a horn without cross-section area reduction, a horn with a neck section located symmetrically in the middle, a horn with a neck located closer to the horn tip, and a horn with a neck located closer to the piezoelectric stack. The five designs of the ultrasonic horn are shown in Figure 6 below for a low frequency actuator we are building for deep drilling.

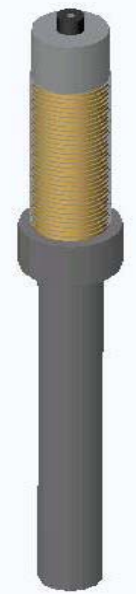

(a)

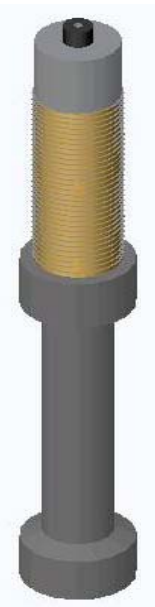

(b)

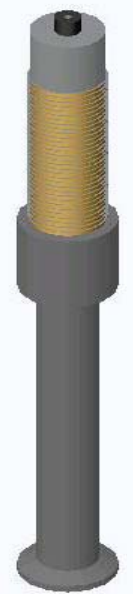

(c)

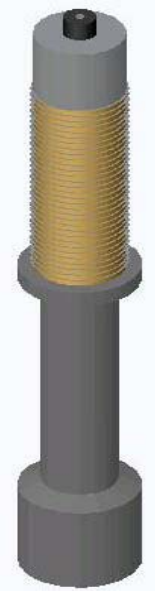

(d)

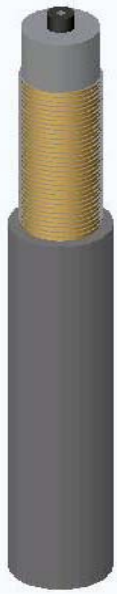

(e)
Figure 6. The 5 designs of ultrasonic horn studied: a) Conventional, b) Neck at middle span of horn, c)Neck moved down $20 \mathrm{~mm}$, d)Neck moved up 20 $\mathrm{mm}$, e) No neck.

Note that the five horns are not of the same scale due to the requirement that the overall length be adjusted to keep the 
neutral plane at the piezoelectric/horn boundary. The actual size of the piezoelectric stack is the same for each horn. A finite element analysis was performed by using the commercially available code ANSYS [6]. In this high power ultrasonic application, the actuator is designed and fabricated to have high mechanical $\mathrm{Q}$, and is operated at or near its first longitudinal resonance frequency. Using modal analysis allowed us to isolate this resonance mode. The anti-resonance frequency of the same mode was derived too, and together with the resonance frequency the electro-mechanical coupling factor was theoretically calculated. The modal analysis is also very useful in determining the neutral plane of the vibration of the actuator.

Table 2. Results of modal and harmonic analysis $(Q=1000)$

\begin{tabular}{|c|c|c|c|c|c|}
\hline $\begin{array}{c}\text { Horn } \\
\text { Type }\end{array}$ & $\begin{array}{c}\text { Horn } \\
\text { length } \\
(\mathrm{mm})\end{array}$ & $\begin{array}{c}\text { Resonance } \\
(\mathrm{Hz})\end{array}$ & $\begin{array}{c}\text { Anti- } \\
\text { Resonance } \\
(\mathrm{Hz})\end{array}$ & $\begin{array}{c}\text { Coupling } \\
\text { Factor }\end{array}$ & $\begin{array}{c}\text { Max. } \\
\text { Displacement } \\
(\mathrm{mm})\end{array}$ \\
\hline Standard & 250 & 5314 & 5726 & 0.372 & 0.209 \\
\hline $\begin{array}{c}\text { Neck at } \\
\text { middle }\end{array}$ & 200 & 5473 & 5947 & 0.391 & 0.185 \\
\hline $\begin{array}{c}\text { Neck up } \\
20 \mathrm{~mm}\end{array}$ & 175 & 5421 & 5916 & 0.400 & 0.185 \\
\hline $\begin{array}{c}\text { Neck } \\
\text { down } 20 \\
\text { mm }\end{array}$ & 255 & 5266 & 5666 & 0.369 & 0.209 \\
\hline $\begin{array}{c}\text { No } \\
\text { Neck }\end{array}$ & 240 & 5304 & 6016 & 0.472 & 0.133 \\
\hline
\end{tabular}

It is desirable to have the neutral plane coincident with the location where the USDC is mounted to the structure of, say, a rover or a robotic arm. Otherwise, the whole structure will interact with the actuator and the interaction may significantly change the design resonance mode of the isolated USDC. Consequently, some energy will be lost dissipating into the whole structure and the efficiency of USDC will go down.

\section{Power VS Momentum (Free Mass 500g)}

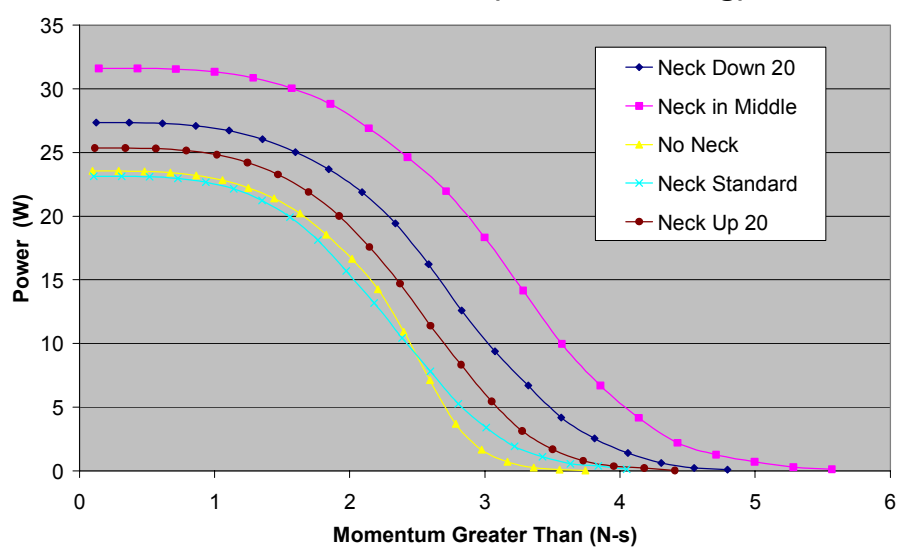

Figure 7. Power as a function of the maximum momentum transfer for the various horn designs.

Experiments show that if the mounting point is not designed to be located at the neutral plane, the mounting does not last and screws or bolts holding the structure to the USDC may thread out or break. Therefore for each design of the ultrasonic horn, the length of the horn is adjusted to align the neutral plane and the mounting point.
After performing the modal analysis a harmonic analysis was performed by exciting the piezoelectric stack with 200-volt peak-to-peak electric field at the resonance frequency. The harmonic analysis provides the maximum horn tip displacement data. The results from the modal and the harmonic analysis are shown in Table 2. The results suggested a specific benefit of the horn with a neck in the middle and the results were then used in a full model of the USDC to determine the power delivered versus the impact momentum delivered to the rock and the results are shown in Figure 7. The results show that both the maximum momentum and the power at each momentum level are greater for the "dog-bone" horn with the neck located symmetrically in the middle.

\section{CONCLUSIONS}

A folded horn was designed and modeled for space applications where reduction of volume and mass is of prime importance. These devices were tested and found to compare favorably to the theory. It was shown that both extensional and bending modes could be added to produce a significant tip displacement and the primary feature controlling this was the fold thickness. In order to increase the drilling efficiency, a "dog-bone" horn was studied to increase the impact momentum of the USDC actuator and preliminary modeling suggest a further increase in the USDC efficiency is possible with this configuration due to the resultant increase in momentum transfer to the bit/rock interface.

\section{ACKNOWLEDGMENT}

The research at the Jet Propulsion Laboratory (JPL), a division of the California Institute of Technology, was carried out under a contract with the National Aeronautics and Space Administration.

\section{REFERENCES}

[1] W.P. Mason, Electromechanical Transducers and Wave Filters, Princeton, NJ, Van Nostrand, 1948

[2] W.P. Mason, Physical Acoustics and the Properties of Solids, D. Van Nostrand Co., Princeton, NJ, 1958

[3]S. Sherrit, B.P. Dolgin, Y. Bar-Cohen, D. Pal, J. Kroh, T. Peterson "Modeling of Horns for Sonic/Ultrasonic Applications," Proceedings of the IEEE International Ultrasonics Symposium, Lake Tahoe, CA, October 1999, pp. 647-651

[4] J. F. Belford, "the Stepped Horn", Proceeding of the National Electronics Conference, 16, Chicago, pp. 814-822, 1960

[5]X. Bao, Y. Bar-Cohen, Z. Cheng, B.P. Dolgin, S. Sherrit, D.S. Pal, S. Du, T. Peterson, "Modeling and Computer Simulation of the Ultrasonic/Sonic Driller/Corer (USDC)", IEEE Transactions on Ultrasonics, Ferroelectrics and Frequency Control, 50, pp. 1147-1160, Sept. 2003

[6]ANSYS Inc. www.ansys.com

[7]S. Sherrit, S.A. Askins, M. Gradziol, B.P. Dolgin, X. Bao, Z. Chang, and Y. Bar-Cohen, "Novel Horn Designs for Ultrasonic/Sonic Cleaning Welding, Soldering, Cutting and Drilling," Paper 4701-34, Proceedings of the SPIE Smart Structures and Materials Symposium, San Diego, CA, March 17-19, 2002 